\title{
Case for dark radiation
}

\author{
Maria Archidiacono, Erminia Calabrese, and Alessandro Melchiorri \\ Physics Department and INFN, Università di Roma "La Sapienza”, Ple Aldo Moro 2, 00185, Rome, Italy
}

(Received 16 September 2011; published 28 December 2011)

\begin{abstract}
Combined analyses of recent cosmological data are showing interesting hints for the presence of an extra relativistic component, coined dark radiation. Here, we perform a new search for dark radiation, parametrizing it with an effective number of relativistic degrees of freedom parameter, $N_{\text {eff }}$. We show that the cosmological data we considered are clearly suggesting the presence of an extra relativistic component with $N_{\text {eff }}=4.08_{-0.68}^{+0.71}$ at $95 \%$ C.L.. Performing an analysis on dark radiation sound speed $c_{\text {eff }}$ and viscosity $c_{\text {vis }}$ parameters, we found $c_{\text {eff }}^{2}=0.312 \pm 0.026$ and $c_{\mathrm{vis}}^{2}=0.29_{-0.16}^{+0.21}$ at $95 \%$ C.L., consistent with the expectations of a relativistic free streaming component $\left(c_{\mathrm{eff}}^{2}=c_{\mathrm{vis}}^{2}=1 / 3\right)$. Assuming the presence of 3 relativistic neutrinos, we constrain the extra relativistic component with $N_{\nu}^{S}=1.10_{-0.72}^{+0.79}$ and $c_{\text {eff }}^{2}=$ $0.24_{-0.13}^{+0.08}$ at $95 \%$ C.L. while $c_{\text {vis }}^{2}$ results as unconstrained. Assuming a massive neutrino component, we obtain further indications for dark radiation with $N_{\nu}^{S}=1.12_{-0.74}^{+0.86}$ at $95 \%$ C.L..
\end{abstract}

DOI: 10.1103/PhysRevD.84.123008

PACS numbers: $98.70 . \mathrm{Vc}$

\section{INTRODUCTION}

Since almost a decade, observations from CMB satellite, balloon-borne and ground-based experiments [1-4], galaxy redshift surveys [5], and luminosity distance measurements are fully confirming the theoretical predictions of the standard $\Lambda \mathrm{CDM}$ cosmological model. This not only permits us to place stringent constraints on the parameters of the model but can be fruitfully used to constrain nonstandard physics at the fundamental level, such as classes of elementary particle models predicting a different radiation content in the Universe.

One of the major theoretical predictions of the standard scenario is the existence of a relativistic energy component (see e.g. [6]), beside CMB photons, with a current energy density given by

$$
\rho_{\text {rad }}=\left[1+\frac{7}{8}\left(\frac{4}{11}\right)^{4 / 3} N_{\text {eff }}\right] \rho_{\gamma}
$$

where $\rho_{\gamma}$ is the energy density of the CMB photons background at temperature $T_{\gamma}=2.728 \mathrm{~K}$ and $N_{\text {eff }}$ is in principle a free parameter, defined as the effective number of relativistic degrees of freedom. Assuming standard electroweak interactions, three active massless neutrinos and including the (small) effect of neutrino flavor oscillations the expected value is $N_{\text {eff }}=3.046$ with a deviation from $N_{\text {eff }}=3$ that takes into account effects from the noninstantaneous neutrino decoupling from the primordial photon-baryon plasma (see e.g. [7]).

In recent years, thanks to the continuous experimental advancements, the value of $N_{\text {eff }}$ has been increasingly constrained from cosmology [1,8-18], ruling out $N_{\text {eff }}=$ 0 at high significance.

However, especially after the new ACT [2] and SPT [4] CMB results, the data seem to suggest values higher than the "standard" one, with $N_{\text {eff }} \sim 4-5$ (see e.g.
$[13,14,17-19])$ in tension with the expected standard value at about 2 standard deviations.

The number of relativistic degrees of freedom obviously depends on the decoupling process of the neutrino background from the primordial plasma. However, a value of $N_{\text {eff }}=4$ is difficult to explain in the three neutrino framework since nonstandard neutrino decoupling is expected to maximally increase this value up to $N_{\text {eff }} \sim 3.12$ (see e.g. [20]). A possible explanation could be the existence of a fourth (or fifth) sterile neutrino. The hypothesis of extra neutrino flavor is interesting since recent results from short-baseline neutrino oscillation data from LSND [21] and MiniBooNE [22] experiments are consistent with a possible fourth (or fifth) sterile neutrino specie (see [13,14] and references therein). Moreover, a larger value for $N_{\text {eff }} \sim 4$ could arise from a completely different physics, related to axions (see e.g. [23]), gravity waves [24], decaying particles (see e.g. [25]), extra dimensions [26,27], and dark energy (see e.g. [28] and references therein).

As a matter of fact, any physical mechanism able to produce extra "dark" radiation produces the same effects on the background expansion of additional neutrinos, yielding a larger value for $N_{\text {eff }}$ from observations.

Since there is a large number of models that could enhance $N_{\text {eff }}$, it is clearly important to investigate the possible ways to discriminate among them. If dark radiation is made of relativistic particles as sterile neutrinos, it should behave as neutrinos also from the point of view of perturbation theory, i.e. if we consider the set of equations that describes perturbations in massless neutrino (following the definition presented in [29]):

$$
\begin{gathered}
\dot{\delta}_{\nu}=\frac{\dot{a}}{a}\left(1-3 c_{\mathrm{eff}}^{2}\right)\left(\delta_{\nu}+3 \frac{\dot{a}}{a} \frac{q_{\nu}}{k}\right)-k\left(q_{\nu}+\frac{2}{3 k} \dot{h}\right), \\
\dot{q}_{\nu}=k c_{\mathrm{eff}}^{2}\left(\delta_{\nu}+3 \frac{\dot{a}}{a} \frac{q_{\nu}}{k}\right)-\frac{\dot{a}}{a} q_{\nu}-\frac{2}{3} k \pi_{\nu},
\end{gathered}
$$




$$
\begin{gathered}
\dot{\pi}_{\nu}=3 c_{\mathrm{vis}}^{2}\left(\frac{2}{5} q_{\nu}+\frac{8}{15} \sigma\right)-\frac{3}{5} k F_{\nu, 3}, \\
\frac{2 l+1}{k} \dot{F}_{\nu, l}-l F_{\nu, l-1}=-(l+1) F_{\nu, l+1}, \quad l \geq 3,
\end{gathered}
$$

it should have an effective sound speed $c_{\text {eff }}$ and a viscosity speed $c_{\text {vis }}$ such that $c_{\text {eff }}^{2}=c_{\text {vis }}^{2}=1 / 3$. Free streaming of relativistic neutrino will indeed produce anisotropies in the neutrino background yielding a value of $c_{\text {vis }}^{2}=1 / 3$ while a smaller value would indicate possible nonstandard interactions (see e.g. [30]). A value of $c_{\text {vis }}$ different from zero, as expected in the standard scenario, has been detected in [31] and confirmed in subsequent papers [32]. More recently, the analysis of [19] confirmed the presence of anisotropies from current cosmological data but also suggested the presence of a lower value for the effective sound speed with $c_{\text {eff }}^{2}=1 / 3$ ruled out at more than 2 standard deviations.

Given the current situation and the experimental hints for $N_{\text {eff }} \sim 4$, it is therefore timely to perform a new analysis for $N_{\text {eff }}$ (and the perturbation parameters $c_{\text {eff }}^{2}$ and $c_{\text {vis }}^{2}$ ) with the most recent cosmological data. This is the kind of analysis we perform in this paper, organizing our work as follows: in Sec. II, we describe the data and the data analysis method adopted. We present our results in the first two subsections of Sec. III, depending on two adopted different parametrizations for the dark radiation. Moreover, a model-independent analysis is also discussed in the last subsection of Sec. III. Finally, we conclude in Sec. IV.

\section{ANALYSIS METHOD}

We perform a COSMOMC [33] analysis combining the following CMB data sets: WMAP7 [1], ACBAR [3], ACT [2], and SPT [4], and we analyze data sets using out to $l_{\max }=3000$. We also include information on dark matter clustering from the galaxy power spectrum extracted from the SDSS-DR7 luminous red galaxy sample [5]. Finally, we impose a prior on the Hubble parameter based on the last Hubble Space Telescope observations [34].

The analysis method we adopt is based on the publicly available Monte Carlo Markov chain package COSMOMC [33] with a convergence diagnostic done through the Gelman and Rubin statistic. We sample the following six-dimensional standard set of cosmological parameters, adopting flat priors on them: the baryon and cold dark matter densities $\Omega_{\mathrm{b}}$ and $\Omega_{\mathrm{c}}$, the ratio of the sound horizon to the angular diameter distance at decoupling $\theta$, the optical depth to reionization $\tau$, the scalar spectral index $n_{S}$, and the overall normalization of the spectrum $A_{S}$ at $k=$ $0.002 \mathrm{Mpc}^{-1}$. We consider purely adiabatic initial conditions and we impose spatial flatness. We vary the effective number of relativistic degrees of freedom $N_{\text {eff }}$, the effective sound speed $c_{\mathrm{eff}}^{2}$, and the viscosity parameter $c_{\mathrm{vis}}^{2}$. In some cases, we consider only variations in the extra dark radiation component $N_{\nu}^{S}=N_{\text {eff }}-3.046$, varying the perturbation parameters $c_{\text {vis }}$ and $c_{\text {eff }}$ only for this extra component and assuming $c_{\mathrm{eff}}^{2}=c_{\mathrm{vis}}^{2}=1 / 3$ for the standard 3 neutrino component.

In our analysis, we always fix the primordial helium abundance to the observed value $Y_{p}=0.24$. This procedure is different from the one adopted, for example, in [4], where the $Y_{p}$ parameter is varied assuming big bang nucleosynthesis for each value of $N_{\text {eff }}$ and $\Omega_{\mathrm{b}}$ in the chain. Since the cosmological epoch and the energy scales probed by $\mathrm{BBN}$ are dramatically different from the ones probed by $\mathrm{CMB}$ and large scale structure, we prefer to not assume standard BBN in our analysis and to leave the primordial helium abundance as fixed to a value consistent with current observations.

We account for foregrounds contributions including three extra amplitudes: the SZ amplitude $A_{\mathrm{SZ}}$, the amplitude of clustered point sources $A_{C}$, and the amplitude of Poisson distributed point sources $A_{P}$. We marginalize the contribution from point sources only for the ACT and SPT data, based on the templates provided by [4]. We quote only one joint amplitude parameter for each component (clustered and Poisson distributed). Instead, the SZ amplitude is obtained fitting the WMAP data with the WMAP own template, while for SPT and ACT it is calculated using the [35] SZ template at $148 \mathrm{GHz}$. Again, this is different from the analysis performed in [4] where no SZ contribution was considered for the WMAP data.

\section{RESULTS}

As stated in the previous section, we perform two different analyses. In the first analysis, we vary the amplitude of the whole relativistic contribution changing $N_{\text {eff }}$ and the corresponding perturbation parameters $c_{\mathrm{vis}}^{2}$ and $c_{\mathrm{eff}}^{2}$. In the second analysis, we assume the existence of a standard neutrino background and vary only the extra component $N_{\nu}^{S}=N_{\text {eff }}-3.046$ considering only in this extra component the variations in $c_{\mathrm{vis}}^{2}$ and $c_{\mathrm{eff}}^{2}$.

\section{A. Varying the number of relativistic degrees of freedom $N_{\text {eff }}$}

In Table I, we report the constraints on the cosmological parameters varying $N_{\text {eff }}$ with and without variations in perturbation theory. We consider two cases: first, we run our analysis fixing the perturbation parameters to the standard values, i.e. $c_{\mathrm{eff}}^{2}=c_{\mathrm{vis}}^{2}=1 / 3$, then we let those parameters vary freely.

As we can see from the results in the left column of Table I, the WMAP7 + ACT + SPT + DR7 + H0 analysis is clearly suggesting the presence of dark radiation with $N_{\text {eff }}=4.08_{-0.68}^{+0.71}$ at $95 \%$ C.L.. When considering variations in the perturbation parameters (right column), the constraint is somewhat shifted toward smaller values with 
TABLE I. MCMC estimation of the cosmological parameters assuming $N_{\text {eff }}$ relativistic neutrinos. Upper bounds at 95\% C.L. are reported for foregrounds parameters. We quote the onedimensional marginalized $68 \%$ and $95 \%$ C.L. for the neutrino parameters.

\begin{tabular}{lcc}
\hline \hline$\Omega_{b} h^{2}$ & $0.02229 \pm 0.00038$ & $0.02206 \pm 0.00081$ \\
$\Omega_{c} h^{2}$ & $0.1333 \pm 0.0086$ & $0.1313 \pm 0.0094$ \\
$\tau$ & $0.082 \pm 0.012$ & $0.083 \pm 0.014$ \\
$H_{0}$ & $74.3 \pm 2.2$ & $74.2 \pm 2.1$ \\
$n_{s}$ & $0.977 \pm 0.011$ & $0.972 \pm 0.021$ \\
$\log \left(10^{10} A_{s}\right)$ & $3.195 \pm 0.035$ & $3.196 \pm 0.035$ \\
$A_{\mathrm{SZ}}$ & $<1.2$ & $<1.4$ \\
$A_{C}\left[\mu \mathrm{K}^{2}\right]$ & $<14.3$ & $<14.6$ \\
$A_{P}\left[\mu \mathrm{K}^{2}\right]$ & $<25.2$ & $<24.7$ \\
$N_{\text {eff }}$ & $4.08_{-0.18-0.68}^{+0.18+0.71}$ & $3.89_{-0.19-0.70}^{+0.19+0.70}$ \\
$c_{\text {eff }}^{2}$ & $1 / 3$ & $0.312_{-0.007-0.026}^{+0.008+0.026}$ \\
$c_{\text {vis }}^{2}$ & $1 / 3$ & $0.29_{-0.06-0.16}^{+0.04+0.21}$ \\
$\chi_{\text {min }}^{2}$ & 7594.2 & 7591.5 \\
\hline \hline
\end{tabular}

$N_{\text {eff }}=3.89_{-0.70}^{+0.70}$. The constraint on the sound speed, $c_{\text {eff }}^{2}=$ $0.312 \pm 0.026$ is fully consistent with the expectations of a free streaming component. Anisotropies in the neutrino background are detected at high statistical significance with $c_{\text {vis }}^{2}=0.29_{-0.16}^{+0.21}$ improving previous constraints presented in [31].

It is interesting to consider the possible degeneracies between $N_{\text {eff }}$ and other "indirect" (i.e. not considered as primary parameters in MCMC runs) model parameters. In Fig. 1, we therefore plot the two-dimensional (2D) likelihood constraints on $N_{\text {eff }}$ versus the Hubble constant $H_{0}$, the age of the Universe $t_{0}$, and the amplitude of rms mass fluctuations on spheres of $8 \mathrm{Mpch}^{-1}, \sigma_{8}$.

As we can see from the three panels in the figure, there is a clear degeneracy between $N_{\text {eff }}$ and those three parameters. Namely, an extra radiation component will bring the cosmological constraints (with respect to the standard 3 neutrino case) to higher values of the Hubble constant and of $\sigma_{8}$ and to lower values of the age of the Universe $t_{0}$. These degeneracies have been already discussed in the literature (see e.g. [36]) and could be useful to estimate the effect of additional data sets on our result. The $3 \%$ determination of the Hubble constant from the analysis of [17] plays a key role in our analysis in shifting the constraints toward larger values of $N_{\text {eff }}$. If future analyses will point toward lower values of the Hubble constant, this will make the standard 3 neutrino case more consistent with observations. If future observations will point toward values of the age of the Universe significantly larger than 13 Gyrs, this will be against an extra dark radiation component, since it prefers $t_{0} \sim 12.5$ Gyrs. Clearly, adding cluster mass function data as presented in [37] that points toward lower values of $\sigma_{8}$ renders the standard $N_{\text {eff }}=$ 3.046 case more consistent with observations. A future and precise determination of $\sigma_{8}$ from clusters or Lyman- $\alpha$ surveys could be crucial in ruling out dark radiation.

\section{B. Varying only the excess in the relativistic component $N_{\nu}^{S}$ and assuming 3 standard neutrinos}

In Table II, we report the constraints considering only an excess $N_{\nu}^{S}$ in the number of relativistic degrees of freedom over a standard 3 neutrinos background.

As we can see from the results in the table, the evidence for an extra background is solid with $N_{\nu}^{S}=1.46_{-0.74}^{+0.76}$ at $95 \%$ C.L. when only variations in the $c_{\text {vis }}^{2}$ component are considered, while the constraint is $N_{\nu}^{S}=1.10_{-0.72}^{+0.79}$ when also variations in $c_{\text {eff }}^{2}$ are considered. Again, the data provide a good determination for $c_{\text {eff }}^{2}$ with $c_{\text {eff }}^{2}=$ $0.24_{-0.13}^{+0.08}$ at $95 \%$ C.L., in marginal agreement at about $2 \sigma$ with the standard $c_{\text {eff }}^{2}=1 / 3$ value. This lower value of $c_{\text {eff }}^{2}$, also found in [19], could hint for a dark radiation component with a varying equation of state, ruling out a
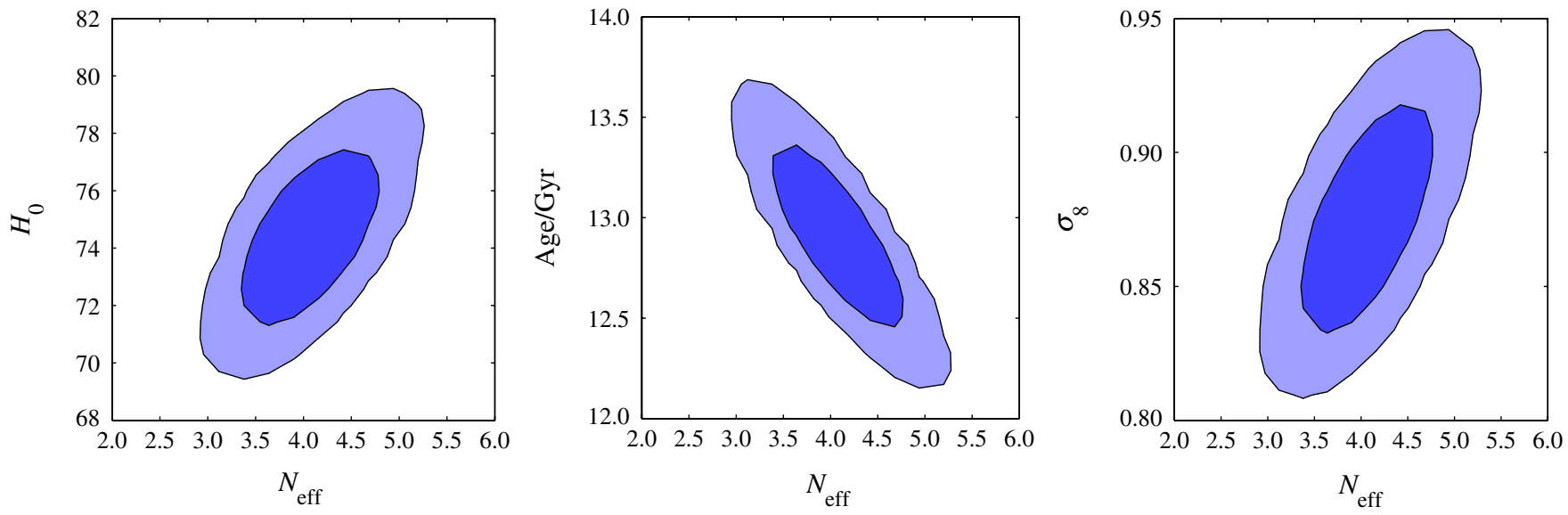

FIG. 1 (color online). $68 \%$ and $95 \%$ C.L. constraints for the degeneracy between $N_{\text {eff }}$ and the Hubble constant $H_{0}$, the age of the Universe $t_{0}$, and the amplitude of mass fluctuations $\sigma_{8}$. 
TABLE II. MCMC estimation of the cosmological parameters considering an extra component $N_{\nu}^{S}$ and assuming a standard background of 3 relativistic neutrinos. The perturbation parameters refer to the extra component. Both $68 \%$ and $95 \%$ confidence levels for the neutrino parameters are reported. Upper bounds are at $95 \%$ C.L..

\begin{tabular}{lcc}
\hline \hline Model: & Varying $c_{\text {eff }}^{2}, c_{\text {vis }}^{2}(\mathrm{~A})$ & $\begin{array}{c}c_{\text {eff }}^{2}=1 / 3, \\
\text { varying } c_{\text {vis }}^{2}(\mathrm{~B})\end{array}$ \\
\hline$\Omega_{b} h^{2}$ & $0.02177 \pm 0.00066$ & $0.02262 \pm 0.00049$ \\
$\Omega_{c} h^{2}$ & $0.135 \pm 0.010$ & $0.143 \pm 0.010$ \\
$\tau$ & $0.086 \pm 0.013$ & $0.084 \pm 0.013$ \\
$H_{0}$ & $72.8 \pm 2.1$ & $73.7 \pm 2.2$ \\
$n_{s}$ & $0.989 \pm 0.014$ & $0.978 \pm 0.014$ \\
$\log \left(10^{10} A_{s}\right)$ & $3.178 \pm 0.035$ & $3.192 \pm 0.035$ \\
$A_{\mathrm{SZ}}$ & $<1.6$ & $<1.4$ \\
$A_{C}\left[\mu \mathrm{K}^{2}\right]$ & $<15.0$ & $<15.0$ \\
$A_{P}\left[\mu \mathrm{K}^{2}\right]$ & $<24.8$ & $<24.8$ \\
$N_{\nu}^{S}$ & $1.10_{-0.23-0.72}^{+0.19+0.79}$ & $1.46_{-0.21-0.74}^{+0.21+0.76}$ \\
$c_{\mathrm{eff}}^{2}$ & $0.24_{-0.02-0.13}^{+0.03+0.08}$ & $1 / 3$ \\
$c_{\mathrm{vis}}^{2}$ & $<0.91$ & $<0.74$ \\
$\chi_{\min }^{2}$ & 7590.5 & 7592.0 \\
\hline \hline
\end{tabular}

massless sterile neutrino. It will be certainly interesting to investigate if this signal remains in future analyses. No significant constraint is obtained on $c_{\mathrm{vis}}^{2}$.

In Fig. 2, we show the degeneracy between the parameters $N_{\nu}^{S}, c_{\text {eff }}^{2}$, and $c_{\text {vis }}^{2}$ by plotting the $2 \mathrm{D}$ likelihood contours between them. As we can see, a degeneracy is present between $c_{\text {eff }}^{2}$ and $N_{\nu}^{S}$ : models with lower values of $N_{\nu}^{S}$ are more compatible with $c_{\text {eff }}^{2}=0$ since the effect of $c_{\text {eff }}^{2}$ on the CMB spectrum is smaller. No apparent degeneracy is present between $c_{\mathrm{vis}}^{2}$ and the remaining parameters since $c_{\text {vis }}^{2}$ is weakly constrained by current data.

Since oscillation experiments have clearly established that neutrinos are massive, it is interesting to perform a similar analysis but let the 3 neutrino standard background with $c_{\mathrm{eff}}^{2}=c_{\mathrm{vis}}^{2}=1 / 3$ be massive, and vary the parameter
TABLE III. MCMC estimation of the cosmological parameters considering $N_{\nu}=3.04$ massive neutrinos. Values and $68 \%-$ $95 \%$ errors for the neutrino parameters are reported. Upper bounds are at $95 \%$ C.L..

\begin{tabular}{lc}
\hline \hline$\Omega_{b} h^{2}$ & $0.02174 \pm 0.00063$ \\
$\Omega_{c} h^{2}$ & $0.135 \pm 0.011$ \\
$\tau$ & $0.087 \pm 0.014$ \\
$H_{0}$ & $72.7 \pm 2.1$ \\
$n_{s}$ & $0.989 \pm 0.015$ \\
$\log \left(10^{10} A_{s}\right)$ & $3.179 \pm 0.036$ \\
$A_{\mathrm{SZ}}$ & $<1.6$ \\
$A_{C}\left[\mu \mathrm{K}^{2}\right]$ & $<15.9$ \\
$A_{P}\left[\mu \mathrm{K}^{2}\right]$ & $<26.1$ \\
$\sum_{\nu} m_{\nu}[\mathrm{eV}]$ & $<0.79$ \\
$N_{\nu}^{S}$ & $1.12_{-0.26-0.74}^{+0.21+0.86}$ \\
$c_{\mathrm{eff}}^{2}$ & $0.241_{-0.02-0.09}^{+0.03+0.09}$ \\
$c_{\text {vis }}^{2}$ & $<0.92$ \\
$\chi_{\text {min }}^{2}$ & 7590.7 \\
\hline \hline
\end{tabular}

$\sum m_{\nu}$ that considers the sum of masses of the 3 active neutrinos. The extra dark radiation component is assumed massless and we treat the perturbations in it as in the previous sections. In Table III, we report the results of this analysis.

As we can see, when masses in the active neutrinos are considered, there is a slightly stronger evidence for the extra background with $N_{v}^{S}=1.12_{-0.74}^{+0.86}$. This can be explained by the degeneracy present between $\sum m_{\nu}$ and $N_{\nu}^{S}$, well known in the literature (see e.g. [13]) and clearly shown in Fig. 3 where we report the 2D marginalized contours in the plane $\sum m_{\nu}-N_{\nu}^{S}$.

\section{Profile likelihood analysis}

Recently, in [38], a model-independent analysis for the extra relativistic degrees of freedom in cosmological data has been performed claiming no statistically significant evidence for it. This simple analysis consists in extracting the maximum likelihood value $L$ as a function of $N_{\text {eff }}$ over
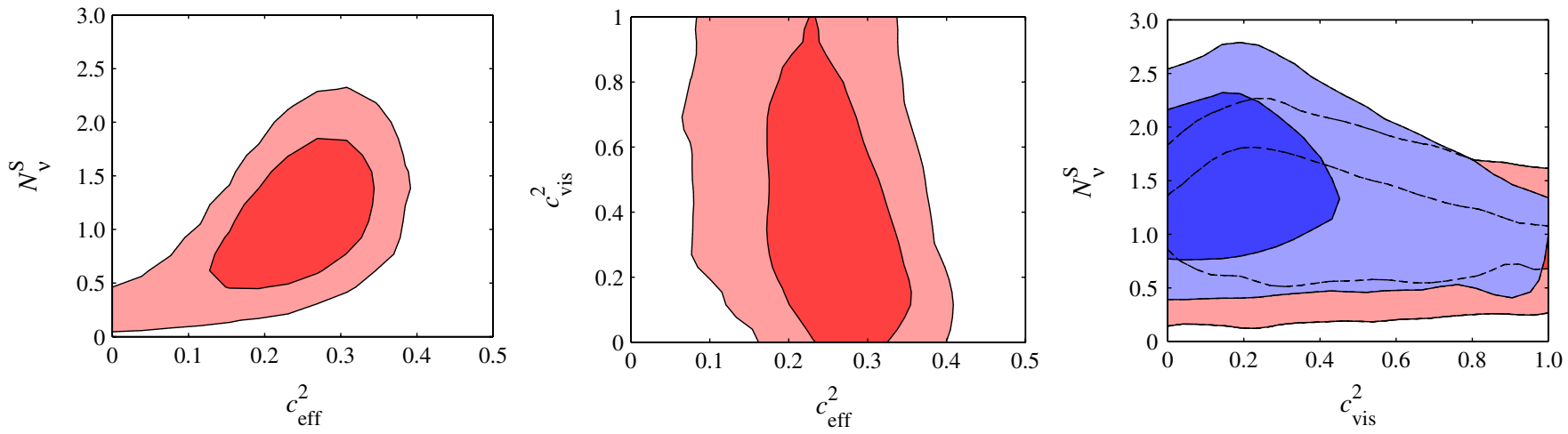

FIG. 2 (color online). $68 \%$ and 95\% C.L. constraints for the degeneracy between neutrinos parameters. Red contours (bottom layer) refer to model (A) in Table II, while blue contours (top layer) show model (B). 


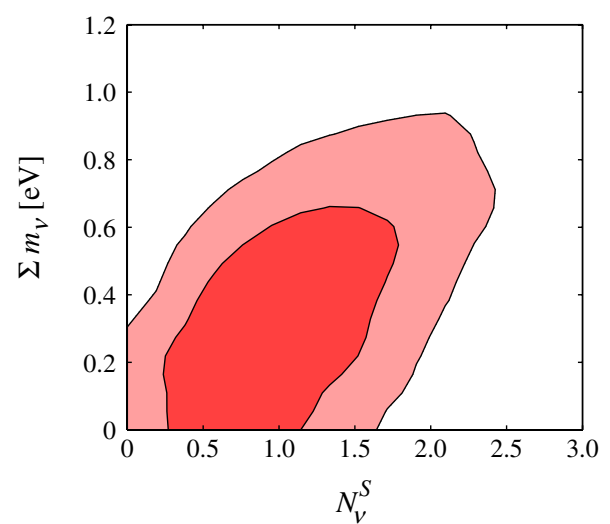

FIG. 3 (color online). Degeneracy in the plane $\sum m_{\nu}-N_{\nu}^{S}$ at $68 \%$ and $95 \%$ C.L..

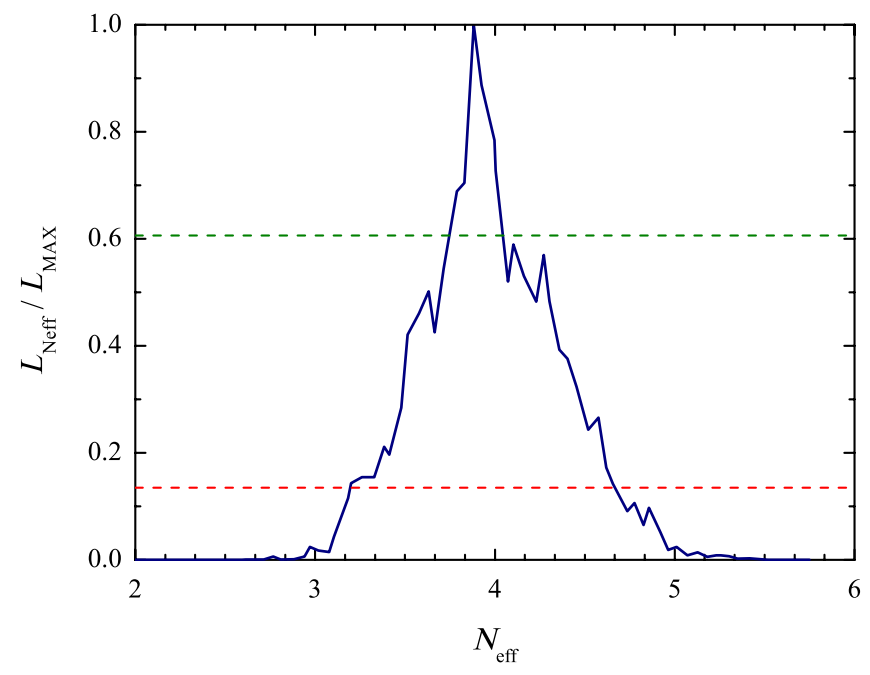

FIG. 4 (color online). Maximum likelihood ratio $L_{N_{\text {eff }}} / L_{\max }$ for $N_{\text {eff }}$. The dashed lines represent the $68 \%$ and $95 \%$ C.L. for a Gaussian likelihood $\left(L_{N_{\text {eff }}} / L_{\max }=0.6065\right.$ and $L_{N_{\text {eff }}} / L_{\max }=0.135$, respectively).

the parameter space sampled in the chains, with a bin width of 0.5 and constructing a profile likelihood ratio by considering $\ln \left(L_{N_{\text {eff }}} / L_{\text {max }}\right)$ as a function of $N_{\text {eff }}$, where $L_{\text {max }}$ is the maximum likelihood in the entire chains.

Here, we perform a similar analysis, using however a smaller bin width of 0.05 and considering the case where the whole number of relativistic degrees of freedom $N_{\text {eff }}$ is varied while $c_{\mathrm{vis}}^{2}=c_{\mathrm{eff}}^{2}=1 / 3$. The resulting likelihood ratio $L_{N_{\text {eff }}} / L_{\max }$, plotted in Fig. 4 , clearly indicates a preference for a dark radiation component finding that the best-fit model has $N_{\text {eff }}=3.88$ with a $\Delta \chi^{2}=14.56$ respect to the best-fit model with $N_{\text {eff }}=3.046$.

We should however point out that the ratio $L_{N_{\text {eff }}} / L_{\max }$ presented in Fig. 4 is rather noisy. Bayesian methods such as MCMC are indeed known to be inaccurate for this purpose (see, for example, the discussion in [39]). Other methods more appropriate for a frequentist analysis have been presented, for example, in [40].

\section{CONCLUSIONS}

In this paper, we performed a new search for dark radiation, parametrizing it with an effective number of relativistic degrees of freedom $N_{\text {eff }}$. We have shown that the cosmological data we considered are clearly suggesting the presence for an extra dark radiation component with $N_{\text {eff }}=4.08_{-0.68}^{+0.71}$ at $95 \%$ C.L.. Performing an analysis on its effective sound speed $c_{\text {eff }}$ and viscosity $c_{\text {vis }}$ parameters, we found $c_{\mathrm{eff}}^{2}=0.312 \pm 0.026$ and $c_{\mathrm{vis}}^{2}=0.29_{-0.16}^{+0.21}$ at $95 \%$ C.L., consistent with the expectations of a relativistic free streaming component $\left(c_{\text {eff }}^{2}=c_{\text {vis }}^{2}=1 / 3\right)$. Assuming the presence of 3 standard relativistic neutrinos, we constrain the extra dark radiation component with $N_{\nu}^{S}=$ $1.10_{-0.72}^{+0.79}$ and $c_{\text {eff }}^{2}=0.24_{-0.13}^{+0.08}$ at $95 \%$ C.L. while $c_{\text {vis }}^{2}$ is practically unconstrained. Assuming a mass in the 3 neutrino component, we obtain further indications for the dark radiation component with $N_{\nu}^{S}=1.12_{-0.74}^{+0.86}$ at 95\% C.L.. From these results, we conclude that dark radiation currently represents one of the most relevant anomaly for the $\Lambda$-CDM scenario.

When comparison is possible, our results are in good agreement with the most recent analysis presented in [19] that uses a different choice of data sets (for example, we do not consider matter fluctuations data from Lyman- $\alpha$ as in [19]) and an independent analysis method.

Dark Radiation will be severely constrained in the very near future by the Planck satellite data, where a precision on $N_{\text {eff }}$ of about $\Delta N_{\text {eff }} \sim 0.2$ is expected (see e.g. [41,42]) only from $\mathrm{CMB}$ data.

\section{ACKNOWLEDGMENTS}

We thank Ryan Keisler for providing us with the likelihood code for the SPT data. We thank Luca Pagano for help. This work is supported by PRIN-INAF, "Astronomy probes fundamental physics." Support was given by the Italian Space Agency through the ASI Contracts "EuclidIC' (I/031/10/0). 
[1] E. Komatsu et al., Astrophys. J. Suppl. Ser. 192, 18 (2011).

[2] J. Dunkley et al., Astrophys. J. 739, 52 (2011).

[3] C. L. Reichardt et al., Astrophys. J. 694, 1200 (2009).

[4] R. Keisler et al., Astrophys. J. 743, 28 (2011).

[5] B. A. Reid et al., Mon. Not. R. Astron. Soc. 404, 60 (2010).

[6] E. W. Kolb and M. S. Turner, Front. Phys. 69, 1 (1990).

[7] G. Mangano, G. Miele, S. Pastor, T. Pinto, O. Pisanti, and P. D. Serpico, Nucl. Phys. B729, 221 (2005).

[8] R. Bowen, S. H. Hansen, A. Melchiorri, J. Silk, and R. Trotta, Mon. Not. R. Astron. Soc. 334, 760 (2002).

[9] U. Seljak, A. Slosar, and P. McDonald, J. Cosmol. Astropart. Phys. 10 (2006) 014.

[10] M. Cirelli and A. Strumia, J. Cosmol. Astropart. Phys. 12 (2006) 013.

[11] G. Mangano, A. Melchiorri, O. Mena, G. Miele, and A. Slosar, J. Cosmol. Astropart. Phys. 03 (2007) 006.

[12] K. Ichikawa, M. Kawasaki, and F. Takahashi, J. Cosmol. Astropart. Phys. 05 (2007) 007.

[13] J. Hamann, S. Hannestad, G. G. Raffelt, I. Tamborra, and Y. Y. Y. Wong, Phys. Rev. Lett. 105, 181301 (2010).

[14] E. Giusarma, M. Corsi, M. Archidiacono, R. de Putter, A. Melchiorri, O. Mena, and S. Pandolfi, Phys. Rev. D 83, 115023 (2011).

[15] L.M. Krauss, C. Lunardini, and C. Smith, arXiv: 1009.4666.

[16] B. A. Reid, L. Verde, R. Jimenez, and O. Mena, J. Cosmol. Astropart. Phys. 01 (2010) 003.

[17] A. G. Riess, L. Macri, S. Casertano, H. Lampeitl, H. C. Ferguson, A. V. Filippenko, S. W. Jha, W. Li et al., Astrophys. J. 730, 119 (2011).

[18] Z. Hou, R. Keisler, L. Knox, M. Millea, and C. Reichardt, arXiv: 1104.2333.

[19] T. L. Smith, S. Das, and O. Zahn, arXiv:1105.3246 [Phys. Rev. D (to be published)].

[20] G. Mangano, G. Miele, S. Pastor, T. Pinto, O. Pisanti, and P. D. Serpico, Nucl. Phys. B756, 100 (2006).

[21] A. Aguilar et al. (LSND Collaboration), Phys. Rev. D 64, 112007 (2001).

[22] A. A. Aguilar-Arevalo et al. (The MiniBooNE Collaboration), Phys. Rev. Lett. 98, 231801 (2007); 103, 111801 (2009).

[23] S. Hannestad, A. Mirizzi, G. G. Raffelt, and Y. Y. Y. Wong, J. Cosmol. Astropart. Phys. 08 (2010) 001; A. Melchiorri, O. Mena, and A. Slosar, Phys. Rev. D 76, 041303 (2007); K. Nakayama, F. Takahashi, and T. T. Yanagida, Phys. Lett. B 697, 275 (2011).

[24] T. L. Smith, E. Pierpaoli, and M. Kamionkowski, Phys. Rev. Lett. 97, 021301 (2006)

[25] W. Fischler and J. Meyers, Phys. Rev. D 83, 063520 (2011); K. Ichikawa, M. Kawasaki, K. Nakayama, M. Senami, and F. Takahashi, J. Cosmol. Astropart. Phys. 05 (2007) 008; K. Nakayama, F. Takahashi, and T. T. Yanagida, Phys. Lett. B 697, 275 (2011).
[26] P. Binetruy, C. Deffayet, U. Ellwanger, and D. Langlois, Phys. Lett. B 477, 285 (2000); T. Shiromizu, K. i. Maeda, and M. Sasaki, Phys. Rev. D 62, 024012 (2000); V. V. Flambaum and E. V. Shuryak, Europhys. Lett. 74, 813 (2006).

[27] A. Hebecker and J. March-Russell, Nucl. Phys. B608, 375 (2001).

[28] E. Calabrese, D. Huterer, E. V. Linder, A. Melchiorri, and L. Pagano, Phys. Rev. D 83, 123504 (2011); J. S. Gagnon and J. Lesgourgues, J. Cosmol. Astropart. Phys. 09 (2011) 026.

[29] W. Hu, Astrophys. J. 506, 485 (1999); W. Hu, D. J. Eisenstein, M. Tegmark, and M. J. White, Phys. Rev. D 59, 023512 (1998).

[30] J. F. Beacom, N. F. Bell, and S. Dodelson, Phys. Rev. Lett. 93, 121302 (2004); S. Hannestad, J. Cosmol. Astropart. Phys. 02 (2005) 011; A. Basboll, O.E. Bjaelde, S. Hannestad, and G. G. Raffelt, Phys. Rev. D 79, 043512 (2009).

[31] R. Trotta and A. Melchiorri, Phys. Rev. Lett. 95, 011305 (2005).

[32] A. Melchiorri and P. Serra, Phys. Rev. D 74, 127301 (2006); F. De Bernardis, L. Pagano, P. Serra, A. Melchiorri, and A. Cooray, J. Cosmol. Astropart. Phys. 06 (2008) 013.

[33] A. Lewis and S. Bridle, Phys. Rev. D 66, 103511 (2002) [http://cosmologist.info].

[34] A. G. Riess, L. Macri, S. Casertano, H. Lampeitl, H. C. Ferguson, A. V. Filippenko, S. W. Jha, W. Li et al., Astrophys. J. 730, 119 (2011).

[35] H. Trac, P. Bode, and J. P. Ostriker, Astrophys. J. 727, 94 (2011).

[36] F. de Bernardis, A. Melchiorri, L. Verde, and R. Jimenez, J. Cosmol. Astropart. Phys. 03 (2008) 020.

[37] A. Vikhlinin et al., Astrophys. J. 692, 1060 (2009).

[38] A. X. Gonzalez-Morales, R. Poltis, B. D. Sherwin, and L. Verde, arXiv: 1106.5052.

[39] Y. Akrami, P. Scott, J. Edsjo, J. Conrad, and L. Bergstrom, J. High Energy Phys. 04 (2010) 057; F. Feroz, K. Cranmer, M. Hobson, R. Ruiz de Austri, and R. Trotta, J. High Energy Phys. 06 (2011) 042.

[40] S. Hannestad, J. Cosmol. Astropart. Phys. 05 (2003) 004; V. Barger, J.P. Kneller, H.-S. Lee, D. Marfatia, and G. Steigman, Phys. Lett. B 566, 8 (2003); P. Crotty, J. Lesgourgues, and S. Pastor, Phys. Rev. D 67, 123005 (2003); K. Ichikawa, M. Kawasaki, and F. Takahashi, J. Cosmol. Astropart. Phys. 05 (2007) 007; J. Hamann, arXiv: 1110.4271.

[41] S. Galli, M. Martinelli, A. Melchiorri, L. Pagano, B. D. Sherwin, and D.N. Spergel, Phys. Rev. D 82, 123504 (2010).

[42] M. Shimon, N. J. Miller, C. T. Kishimoto, C. J. Smith, G. M. Fuller, and B. G. Keating, J. Cosmol. Astropart. Phys. 05 (2010) 037. 